\title{
20 Jahre Deutsche Krebshilfe e. V.
}

Frau Dr. med. Mildred Scheel gründete 1974 als Frau des damaligen Bundespräsidenten, Dr. Walter Scheel, die Deutsche Krebshilfe e.V. Mit dieser Bürgerinitiative verfolgte Frau Dr. Scheel vornehmlich drei Ziele:

- Krebs aus der Tabuzone des Schweigens zu holen und als behandelbare Krankheit in das Bewußtsein der Allgemeinheit zu heben.

- Die fachliche Koordination und interdisziplinäre Zusammenarbeit bei der Behandlung krebskranker Menschen zu verbessern.

- Eine effektive Krebsbekämpfung durch eine weitreichende und langfristige Krebsforschung zu unterstützen.

Um diese drei Hauptziele zu verwirklichen, wandte sich Frau Dr. Scheel über die Medien direkt an die Menschen und bat um idielle und materielle Unterstützung. Zur Förderung der interdisziplinären Zusammenarbeit wurde auf Anregung von Fachleuten im Jahre 1976 das erste Tumorzentrum in der Bundesrepublik Deutschland in Hamburg gegründet, dem in rascher Folge die Gründungen in Köln und München folgten. Dieser Gedanke hat sich als so effektiv erwiesen, daß nunmehr ein flächendeckendes Netz von Tumorzentren und onkologischen Schwerpunkten in der Bundesrepublik besteht. Zur langfristigen Forschungsförderung wurde 1976 die Dr. Mildred Scheel Stiftung gegründet, durch die eine langfristige Grundlagenforschung, internationale Expertentreffen und Stipendienprogramme für junge Wissenschaftler ermöglicht werden.

Obwohl Frau Dr. Scheel als Röntgenologin mit der Krankheit Krebs beruflich täglich konfrontiert war und die tagtäglich zu erfahrenden Unzulänglichkeiten die Hauptmotivation für ihr ungewöhnliches Engagement darstellten, wurde die Arbeit der Deutschen Krebshilfe e. V. anfänglich von den Krebsspezialisten und Verbänden nicht ohne Argwohn begleitet. Deshalb war es eine konsequente Entscheidung, daß den Vorständen der Deutschen Krebshilfe und der Dr. Mildred Scheel Stiftung Beratungsgremien zur Seite gestellt wurden, in die in der Krebsbehandlung erfahrene und anerkannte Ärzte sowie in der Krebsforschung ausgewiesene Wissenschaftler ihren Sachverstand einbrachten.

Schon früh wandte sich die Deutsche Krebshilfe e. V. der Behandlung krebskranker Kinder und Jugendlicher zu und gründete hierfür speziell die Kinderkrebshilfe. In den zwanzig Jahren des Bestehens der Deutschen Krebshilfe sind mehr als $70 \mathrm{Mio}$. DM für dieses Förderungsziel bereitgestellt worden, das sind rund $10 \%$ des gesamten Fördervolumens der Deutschen Krebshilfe in dieser Zeit.

Die Förderung durch die Kinderkrebshilfe ist vielfältig und breit gefächert. Anfänglich war die Beseitigung von

Klin. Pädiatr. 206 (1994) 189-190

(C) 1994 F. Enke Verlag Stuttgart
Notsituationen bei der ambulanten und stationären $\mathrm{Be}$ handlung krebskranker Kinder und Jugendlicher eine Hauptaufgabe. Die Deutsche Arbeitsgemeinschaft für Leukämieforschung und -behandlung im Kindesalter e.V. (gegründet 1965) und die Gesellschaft für Pädiatrische Onkologie und Hämatologie (gegründet 1974) hatten kooperative Behandlungspläne aufgestellt, die zu erhöhten Heilungschancen bei krebskranken Kindern und Jugendlichen führten. Da diese Behandlungen vorzugsweise in den Universitätskliniken durchgeführt wurden, nahm die Zahl der dort zu behandelnden Kinder rasch $\mathrm{zu}$, ohne daß die Kliniken auf diese neue Aufgabe von langer Hand vorbereitet waren. So war es nicht verwunderlich, daß die stationäre und ambulante Behandlung für die um ihr Leben kämpfenden Kinder von personellen und organisatorischen Engpaßsituationen gekennzeichnet war. Durch die schwerpunktmäßige Hilfe und Unterstützung wurden Notsituationen bereinigt, aber auch die Krankenhäuser und die Landesregierungen auf ihre Verpflichtung gegenüber dieser Patientengruppe hingewiesen.

In einer gemeinsamen Aktion von ZDF, Stern und Deutscher Krebshilfe e.V. wurde speziell um Spenden für die Kinderkrebshilfe geworben. Mit den erhaltenen Spenden wurde die Einrichtung von Elternhäusern und -wohnungen in Kliniknähe unterstützt. Zur schonenderen und besseren Diagnostik wurden hochauflösende Computersonographiegeräte zur Verfügung gestellt, die heute aus dem Kliniksalltag nicht mehr wegzudenken sind. Diese Förderungsmaßnahme wurde auch nach der 1989 erfolgten Vereinigung der beiden Teile Deutschlands auf die neuen Bundesländer ausgedehnt. Unterstützt und überbrückt wurde weiterhin der Aufbau der psychosozialen Betreuung der krebskranken Kinder und ihrer Familien. Auch die Nachsorgekliniken, die sich auf die Ausrichtung von familiengerechten Kuren für krebskranke Kinder und Jugendliche spezialisiert haben, wurden in den Förderungsrahmen mit aufgenommen.

Mit dem Auslaufen des Krebsprogrammes der Bundesregierung hat sich die Deutsche Krebshilfe e. V. die Weiterförderung der pädiatrischen Therapiestudien zu einem besonderen Anliegen gemacht. Durch die kontinuierliche Verbesserung der kooperativen Protokolle zur Diagnostik und Therapie gelingt es, daß mehr als zwei Drittel der erkrankten Kinder und Jugendlichen überleben. Diesen hohen, flächendeckenden Qualitätsstandard gilt es nunmehr langfristig abzusichern, was mit einer weiterführenden Hilfe der Deutschen Krebshilfe für die nahe Zukunft erhofft wird.

Der Prävention und Früherkennung von bösartigen Erkrankungen fühlt sich die Deutsche Krebshilfe e.V. als Bürgerinitiative besonders verpflichtet und unterstützt die Kommission zur Früherkennung und Vermeidung von Hautkrebs sowie das Pilotprogramm zur Früherkennung von Neuroblastomen. 
Fortbildung für Schwestern und Ärzte werden durch Seminare und Stipendien seit vielen Jahren gefördert und haben zum jetzigen Qualitätsstandard einen erheblichen Beitrag geleistet. Die Förderung der Grundlagenforschung auf zell- und molekularbiologischer Ebene, Tumorimmunologie und Erkennung von Risikofaktoren müssen zukünftig noch stärkere Berücksichtigung in der Forschungsförderung finden, ebenso wie die Früherkennung von Späteffekten einer erfolgreichen Krebsbehandlung.

Die regionale Hyperthermie als Ergänzung von Operation, Bestrahlung und Chemotherapie bei der Behandlung bösartiger Tumoren und die Knochenmarktransplantation bei systemischen Erkrankungen werden schwerpunktmäßig als innovative Behandlungsverfahren gefördert. Hier ist vor allem auch der Aufbau der Deutschen Knochenmarkspenderdatei eine Maßnahme von weitreichender Wirkung, von der vor allem Kinder mit akuten Leukämien profitieren. Wünschenswert ist die Ergänzung der bisherigen serologischen Klasse I Typisierungen durch eine molekularbiologische Klasse II Typisierung, um die Suchzeiten für die wartenden Patienten weiter abzukürzen. Gleichzeitig ist jedoch auch eine Erhöhung der Transplantationskapazität durch Ausbau der bisherigen Transplantationseinrichtungen und Gründung neuer Transplantationszentren erforderlich, um den Bedürfnissen der Patienten und den geschaffenen Möglichkeiten gerecht zu werden.

So verbinden sich der Dank und die Anerkennung für die Leistungen der Deutschen Krebshilfe e.V. während der vergangenen Jahre unvermeidbar auch mit Wünschen und Hoffnungen an die Zukunft. Mag die Äußerung von Wünschen vor dem Hintergrund einer umfangreichen Förderung über 20 Jahre ungewöhnlich sein, so wird hierdurch aber die Notwendigkeit für weitere Hilfen und Förderungen offensichtlich.

Wir gratulieren der Deutschen Krebshilfe e. V. zu ihrer $20 \mathrm{jährigen} \mathrm{erfolgreichen} \mathrm{Tätigkeit} \mathrm{und} \mathrm{wünschen} \mathrm{ihr}$ weiter wachsende Anerkennung und Unterstützung durch die Öffentlichkeit, um ihre selbstgestellten Aufgaben zum Wohle der Krebskranken erfüllen zu können.

\section{Prof. Dr. Kurt Winkler}

Vorsitzender der Gesellschaft für

Pädiatrische Onkologie und Hämatologie

Universitäts-Kinderklinik

Martinistr. 52

D.20246 Hamburg-Eppendorf 